\title{
Metallic and intra-band investigation of optical properties for Borophene nano-sheet: a DFT study
}

\author{
T. Abasi ${ }^{1}$ - A. Boochani ${ }^{2}$ 'S. R. Masharian ${ }^{1}$
}

Received: 26 July 2019 / Accepted: 31 October 2019 / Published online: 14 November 2019

(c) The Author(s) 2019

\begin{abstract}
In this paper, using the density functional theory framework with the FP-LAPW + lo method by GGA approximation, the electronic and optical properties such as band structure, density of states, dielectric function, energy loss function, absorption and reflection have been investigated for borophene nano-sheet. The optical properties of the borophene have been changed as the incident light direction whereas has the metallic and semiconductor behavior, in the borophene sheet and perpendicular light angles, respectively. Therefore, it can be said that the optical properties of this material are anisotropic.
\end{abstract}

Keywords DFT $\cdot$ Borophene $\cdot$ Electronic properties $\cdot$ Optical properties

\section{Introduction}

After discovery of graphene, researches have been carried out to discover two-dimensional allotropes of other elements. Some of the most important features of graphene are mechanical hardness, high thermal and electrical conductivity, high density, and charge mobility. Charge carriers in graphene behave like Dirac massless fermions [1] so that in addition to the high mobility of charge carriers, it absorbs only $2.3 \%$ of visible light [2], so graphene is a suitable candidate to use in transparent conductors.

The other two-dimensional allotropes of the elements of group IV are silicene [3-5], germanene [6-8] and stanene [9-11] which are predicted to be stable and are synthesized using molecular beam epitaxy (MBE) growth in high vacuum conditions. With the boom in research in the field of two-dimensional materials, scientists predicted that the neighbor of carbon atom in the periodic table, boron, could also be arranged and ordered as a single atomic surface. Due to the interesting physical and chemical properties of boron, such as exceptional structural complexity, extreme hardness, superconductivity and high chemical stability, it

$\triangle$ A. Boochani

arash_bch@yahoo.com

1 Department of Physics, Hamedan Branch, Islamic Azad University, Hamedan, Iran

2 Department of Physics, Kermanshah Branch, Islamic Azad University, Kermanshah, Iran has attracted a lot of attention [12]. Boron is the only nonmetallic substance in the group III which has more than 16 structures with interesting properties $[16,17]$. One of the interesting features of boron is the $\mathrm{B}_{12}$ twin-cages that is the base in the bulk and many of its compounds [12, 15]. As well as boron clusters that are predicted to be in the form of a planar or quasi-planar such as $\mathrm{B}_{36}$ and $\mathrm{B}_{19}{ }^{-}, \mathrm{B}_{13}{ }^{+}$, and $\mathrm{B}_{12}{ }^{-}$while the base of the neighbor atom of boron in the bulk (graphite) is in the form of a two-dimensional surface of carbon atoms and its clusters are in the form of fullerenes [13, 16-18].

In the recent years, two-dimensional boron surfaces have been studied theoretically [19-21] and experimentally [22, 23]. Penev et al. [19] and Wu et al. [20] studied the stability and electronic properties of the boron free single-layer with different structures. They identified five different types of boron single-layer based on the structure and number of neighbor atoms (neighbor numbers) of boron atom, and also they predicted the most stable types of layer by obtaining the adhesion energy value and the layers forms. In addition, they also investigated the metallic and semiconductor properties and the energy gap of different structures by studying the densities of states and band structures. Theoretically, a large number of two-dimensional flat boron structures are predicted with different adhesion energy, such as $\alpha$ layers, $\beta$ layers and $\gamma$ layers $[24,25]$. Boron two-dimensional layers are composed of rows of boron atoms formed by hexagonal holes. In the case of two-dimensional structures, a parameter, $\eta$, is defined as the ratio of the number of the hexagonal 
holes to the number of atoms in the primary unit cell, which, according to the way in which the atoms are placed and the different shape of the hexagonal holes, this parameter changes and causes the different layers.

Several studies have been carried out using the first principle calculations to study the various properties and stable structures of borophene [26] which these studies have resulted in an appropriate viewpoint on applied issues. The $\mathrm{X}$-ray photoelectron spectroscopy (XPS) study showed that boron two-dimensional structures are completely stable against oxidation, and this increased the attractiveness of these structures for further investigation for practical applications in the industry [23]. Scanning tunneling spectroscopy measurements have shown that borophene has metallic properties while its bulk state is semiconductor [22]. Experimentally, Mannix et al. [22] fabricated a two-dimensional boron sheet, called borophene, in a high vacuum condition on the substrate of $\mathrm{Ag}$ (111) and described its metallic properties. Meanwhile, Feng et al. [23] synthesized the borophene structure on the Ag (111) substrate using molecular beam epitaxy (MBE) method under UHV conditions. They believed that borophene contained flat atomic structures including triangular grids with a different order from hexagonal holes, called $\beta_{12}$ and $\chi_{3}$ boron nano-sheets.

One of the important factors for identifying the materials applications is the investigation of their electronic and optical properties to the external phenomena such as electric current and optical beam. In this paper, the density functional theory is used to study the electronic and optical properties of borophene nano sheet with the Pmmm space group. This paper has been organized as follow that first, the calculation details are discussed, and then, the electron properties is investigate by studying the density of states diagram and band structure. Finally, the optical properties are examined using the optical diagrams.

\section{Computational details}

The first principle calculations were performed based on the density functional theory and the FP-LAPW + lo model using the Wien $2 \mathrm{~K}$ simulation package and the generalized gradient approximation (GGA) is also used for the exchange-correlation energy. The muffin-tin radius for boron is obtained $1.44 \AA$, and the other optimized input parameters such as RKmax, Kpoint, lmax and Gmax are selected to 8.0, 500, 10 and 12, respectively. Moreover, to obtain the relaxed structure, the atomic forces have been converged to $0.1 \mathrm{mRyd} / \mathrm{a}$.u. At the end, the optical calculations have been approximated by RPA [27]. The cohesive energy of this compound has been calculated to $-3.78 \mathrm{eV}$ which is referred to its stability.

\section{Results and discussion}

\section{Structural and electronic properties}

More than 10 types of borophene sheets are predicted theoretically [36] and experimentally [22, 23] which the differences in their structures lays are in the regular pattern of the removed boron atoms in the unit cell. The figure below depicts the borophene relaxed structure with the pmmm space group. The plane sheet contains periodic rows of boron atoms with hexagonal cavities which is one of the different structures of borophene that has been theoretically investigated. There are three atoms per unit cell, and the optimized lattice constants are $a=2.87 \AA, b=3.25 \AA$ and $c=12.99 \AA$ which are in a good agreement with the reported values [28] (Fig. 1).

The density of states provides us with very useful information about the electronic and optical features. The total and partial densities of states of borophene composition are shown in Figs. 2 and 3. The density of states indicates the number of allowed states per unit of energy, and it can be used to express the orbital contribution of the participating atoms. According to the graphs, the density of states is nonzero at Fermi energy, and the valence and conduction bands are overlapping at Fermi level. The energy gap is zero, so it has a metallic property which is also confirmed by band structure. Unlike many two-dimensional materials acting as semiconductor or semimetal, borophene has a metallic property that has been reported in other papers [22, 23, 29-33].

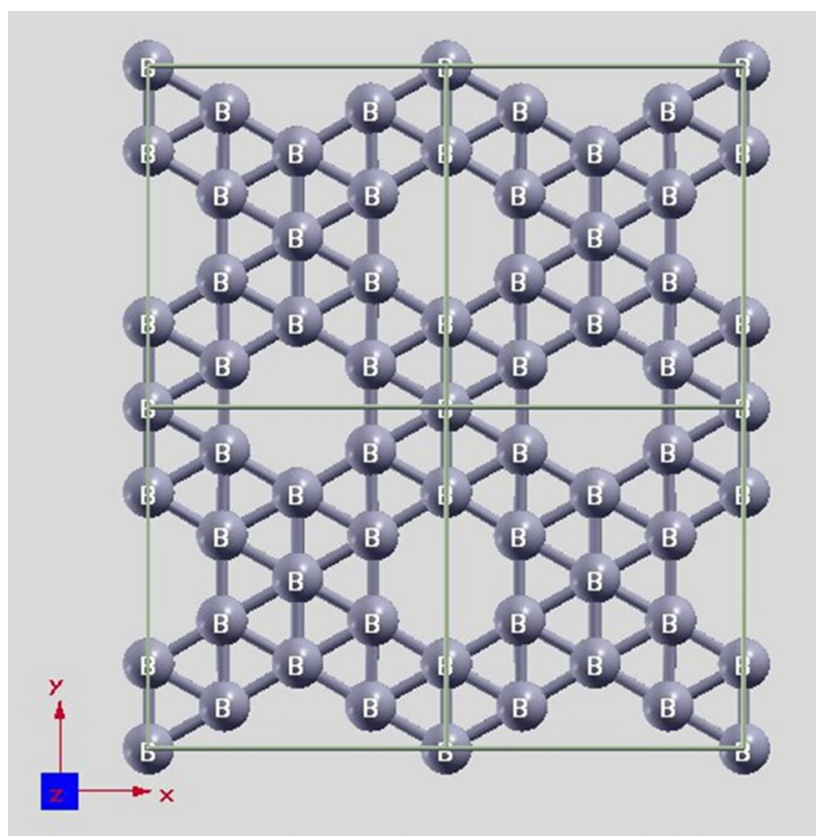

Fig. 1 Two-dimensional boron with pmmm space group 


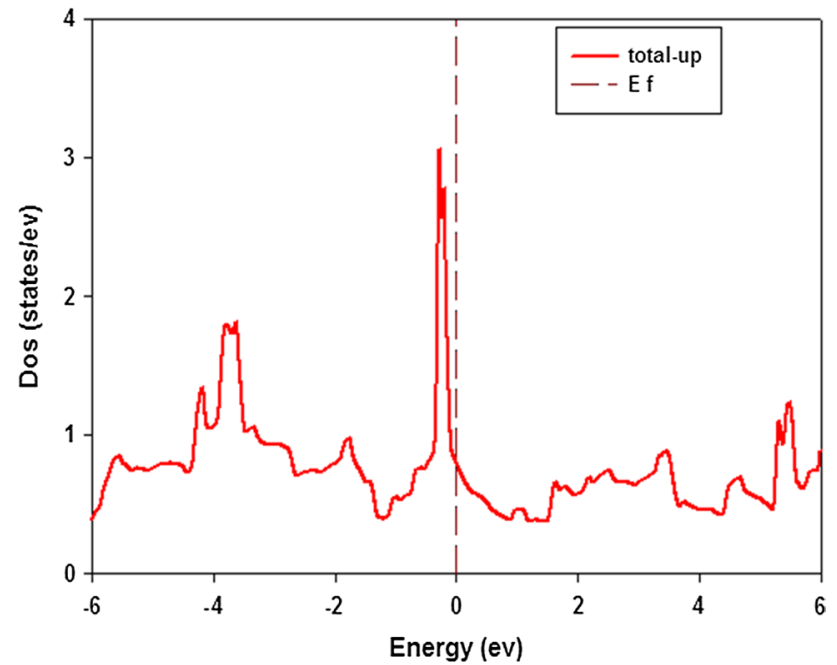

Fig. 2 Density of states (Dos) for pmmm boron. The Fermi level is set to energy zero

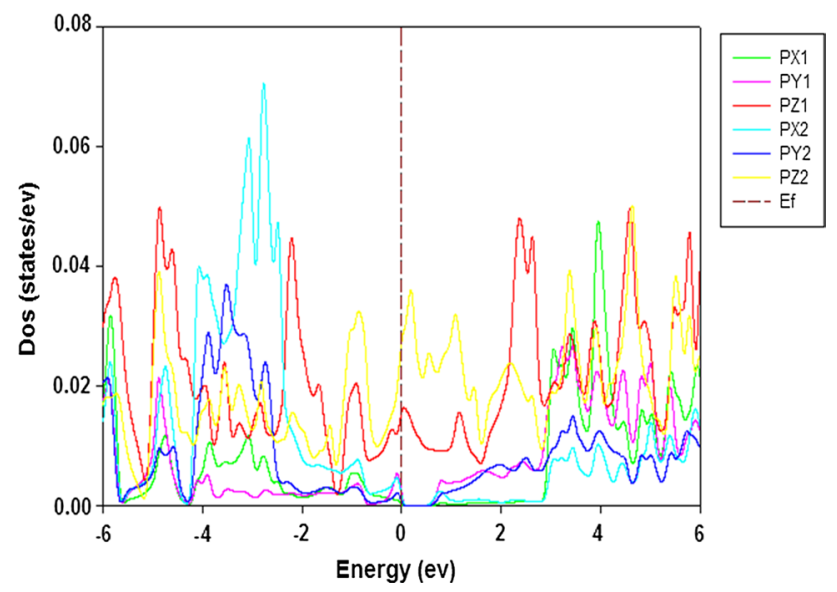

Fig. 3 Partial Density Of State (PDOS) for pmmm boron. The Fermi level is set to energy zero

The continuity DOS means that it is a very good electron conducting compound.

In the density of states, zero energy represents Fermi level, and the distribution of orbitals around the Fermi level is high. In the vicinity of the Fermi level, the orbitals contribution in the valence band is greater than their contribution in the conduction band. Since the density of states for the up and down spins is symmetrical, the compound shows no magnetic properties. In the band structure diagram, levels also cut off Fermi level confirming the metallic property of the structure. By comparing the density of states and the band structure diagrams, a good agreement between them can be observed.

The densities of states at Fermi level belong to the $\mathrm{p}$ orbital of the boron atom which its s orbital does not play any role at Fermi level. As shown in Fig. 2, the densities of states, especially in the conduction region, are turned into a sawtooth due to the nanometer dimensions of this composition, which is also confirmed in Fig. 3 for each atom. Another important point to note here is that the borophene composition has a very good metallic behavior because the densities of states are completely continuous from the valence region about $-5 \mathrm{eV}$ to the conduction region and higher energies, and no energy gap can be detected. So, if an electron is transferred to the region for any reason, high conductivity would be expected. In the Fig. 3, it is shown that the Pz2 and Pz1 degeneracies of the B atoms have the main role in the Fermi level and also, the Px2 has more contribution in the valance bond. The gradients of the band structure diagram at the symmetric points of $R-\Gamma$ and $X-M$ and $M-\Gamma$ confirm the above discussion, so that the proper gradient of the diagrams, whether at Fermi region, or in the valence and conduction regions, is a good evidence of high conductivity. The slope of the energy band indicates the group velocity, and its arc represents the effective mass.

It is clear from the partial density of states diagram that the $\mathrm{p}$ orbital cuts Fermi level, and given that the s orbital contribution is very small, so it does not have a significant contribution to the metallic property and the main contribution is for the $\mathrm{p}$ orbital. The major contribution of the $\mathrm{s}$ orbital is above the conduction band. In general, the contribution of the $\mathrm{p}$ orbital is greater than that of the s orbital, and accordingly, this orbital has a greater contribution in the electronic conduction. The bandstructure of this nano-sheet in Fig. 4 has been shown that the steep slopes of the levels along the $\mathrm{M}$ symmetry direction are higher than other directions which referred to the high mobility of the electrons and holes and low their effective mass. Based on the high amount of the DOS at Fermi level and steep slope of the two levels

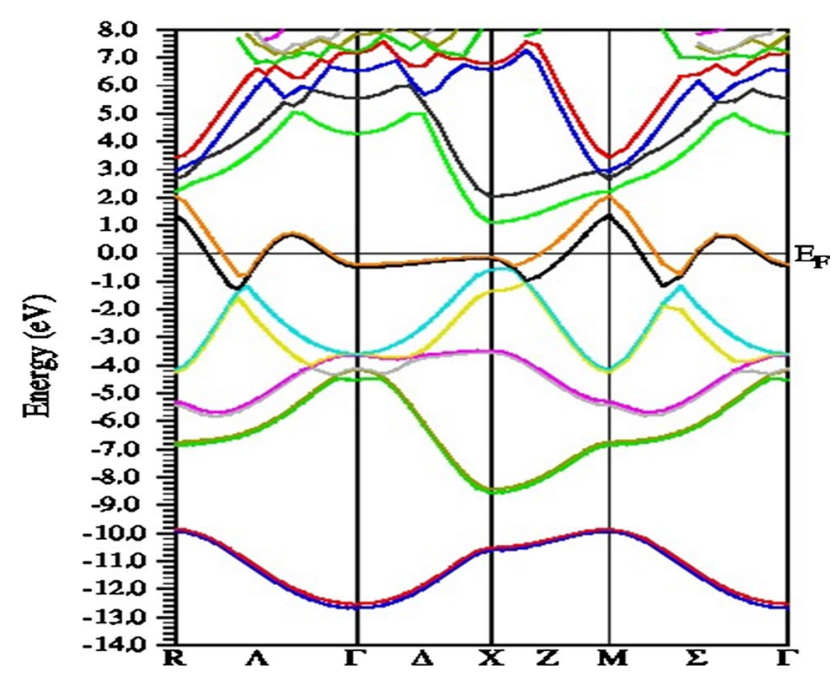

Fig. 4 Electronic band structure of pmmm boron 
that cut the Fermi along the M direction in the Fig. 4, in this nano-sheet the electron mobility's are high and is a good candidate for transport applications.

\section{Optical Properties}

The optical properties of the materials obtain from the Kramars-Kroing equations which are based on the dielectric tensor including the real and imaginary parts as bellow:

$\varepsilon(\omega)=\varepsilon_{1}(\omega)+i \varepsilon_{2}(\omega)$,

The real and imaginary parts of the dielectric function are shown in Fig. 5 in two directions of the perpendicular $(z)$ and parallel to the sheet $(x)$, which is used to describe the crystal response to the electromagnetic field, and depends on the electronic band structure of the crystal. The dielectric function is the matter response to the electromagnetic waves, showing energy transfer, absorption and energy loss. That is, wherever a part of the dielectric function is negative, electromagnetic waves become weak and absorption or reflection occurs. The real and imaginary parts of the dielectric function are related by the so-called Kramers-Kroing relations, and it possible to determine the real part of the response optical function with knowledge of the imaginary part at all frequencies and vice versa. The dielectric function has two interband and intraband transitions. The interband transition is due to the absorption edges excitation and the intraband transition is due to the volume Plasmon excitation. The imaginary part of the dielectric function is calculated by taking into account all possible transitions from occupied to unoccupied states. In Fig. 5a, the static value of the real part has shown that its value is 3.60 in the $\mathrm{z}$ direction, and its value has shifted to the negative infinite along the $\mathrm{x}$ direction. Therefore, by changing the direction of the incident light to this structure, it is expected to detect almost semiconductor behavior in the $z$ direction (perpendicular to the plane) and severe metallic behavior along the $\mathrm{x}$ direction (on the plane). By increasing the incident photon energy, there is a sharp decrease at the edge of the visible region for the real part to reach its value to zero, but the response increases with a mild slope afterward to $7.8 \mathrm{eV}$. Therefore, the continuity of this response leads to a completely stable and balanced optical response to the light within the mentioned range for borophene. The optical responses along $x$ and $z$ axes are constantly zero after $10.68 \mathrm{eV}$ and less than one for higher energies, so borophene optically behaves like a vacuum environment after $14.1 \mathrm{eV}$ in both directions. In the $\mathrm{x}$ direction, owing to the extremely severe metallic behavior before $7.2 \mathrm{eV}$, while the real part is negative, it becomes continuous zero after some fluctuations, but it acts the same as the $z$ direction after $12.27 \mathrm{eV}$.

According to the imaginary part of the dielectric function (Fig. $5 b$ ), it can be seen that the imaginary part value is 0.3 at zero energy for EllZ mode indicating semiconductor behavior while it tends to positive infinite for EllX representing the extremely intense metallic behavior. The peaks of the imaginary part demonstrate the interband transitions. For the EllX mode, two peaks are observed at energies of $7.45 \mathrm{eV}$ and $2.58 \mathrm{eV}$ illustrating the interband transitions in turn from the Px2 orbital to the Pz2 orbital and from the Pz1 orbital to the Pz2 orbital. For EllZ mode, there are also two peaks at energies of $0.78 \mathrm{eV}$ and $8.62 \mathrm{eV}$ indicating the interband transition from the Pz1 orbital to the Pz2 orbital and from Px 2 orbital to the Pz2 one. In fact, each peak in the diagram of the imaginary part of the dielectric function can be assigned to an interband transition. The peaks of the
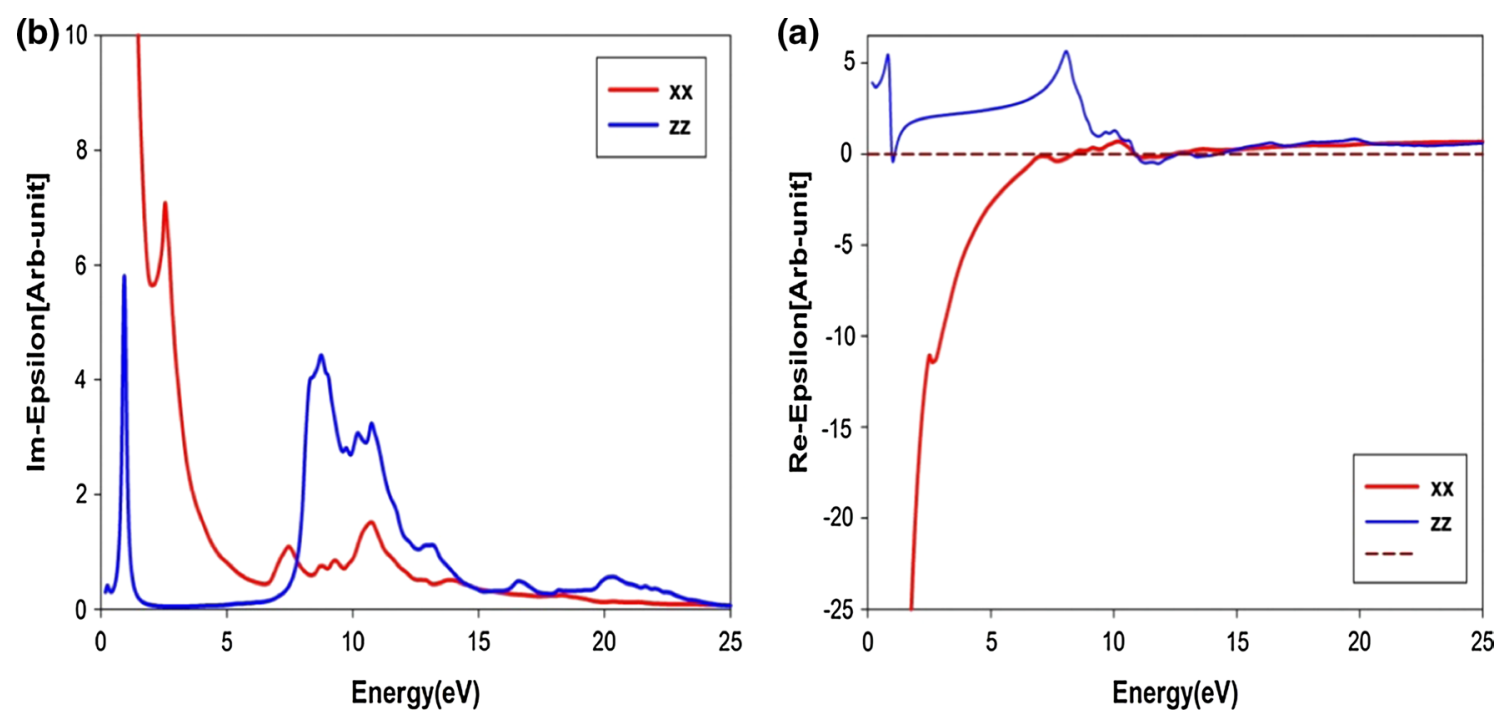

Fig. 5 The a real part and $\mathbf{b}$ imaginary part of the dielectric function for pmmm boron 
imaginary part of the dielectric function and the ELOSS function represent the Plasmon oscillations, which are listed for the two directions in Table 1. At energies of more than $10 \mathrm{eV}$, the dielectric function behavior is the same for both ElIZ and ElIX. The real and imaginary parts of the dielectric function behave as anisotropic. No transition occurs along the $z$ axis from the visible range to about $8 \mathrm{eV}$ while there is a steep slope along the $x$ axis. However, some peaks exist from 7 to $15 \mathrm{eV}$ in both directions illustrating interband transitions; the amount of transitions practically reaches its minimum after $15 \mathrm{eV}$ expressing that the real part behavior is completely in line with the imaginary part behavior. The Dirac form of the imaginary part of dielectric function along the $z z$ direction in the IR region indicated the instability of optical absorption.

One of the important parameters in optics is the energy loss function. There are several ways to stimulate the electrons in a solid. One way that depends on the optical spectrum is the excitation of electrons by other electrons, which is done by illuminating a sample with a single-energy electron beam and decomposing reflected energy. The energy dissipation spectrum can be due to the excitation of singleenergy electrons in the solid occurring by photon absorption, or from the collective stimulation of valence electrons, called Plasmon, or from any other excitation. The energy loss spectrum of structure is shown in Fig. 6 in which peaks are related to interband and intraband transitions. The most significant peaks in the diagram are at energies of $1.05 \mathrm{eV}$ and $12.48 \mathrm{eV}$ and $14.43 \mathrm{eV}$ for the EllZ mode, and at energies of $6.87 \mathrm{eV}, 8.35 \mathrm{eV}, 12.53 \mathrm{eV}$ and $13.06 \mathrm{eV}$ for the EllX one demonstrating the Plasmon oscillations and the collective excitation of the electrons. The behaviors of the loss function along $x$ and $z$ indicate anisotropy. In the Ell $x$ mode, there are no loss or very small in the IR region and UV energy. However, in the Ell $z$ case, we have a Dirac peak in the visible edge after which there is a gap till $7.5 \mathrm{eV}$. This sharp peak suggests instability in optical behavior that is due to zeroing the real part of the dielectric function at this energy. Therefore, the best optical response in both directions for this composition goes back to the IR (visible) region and the low UV, so that an intense increase in the peaks can be seen at higher UV energies (After $5 \mathrm{eV}$ ).

The refractive index is a complex quantity in which the real part is the conventional refractive index of the material, and its imaginary part is called the extinction coefficient, which is directly related to the material absorption. According to Fig. 7, the static refractive index for the ElIZ state is 1.98 , and its maximum value is 2.43 at the energy of $0.68 \mathrm{eV}$ while its minimum is 0.82 at the energy of $1 \mathrm{eV}$. For the EllX mode, the refractive index decreases from its maximum value to reach its minimum at the energy of $6.2 \mathrm{eV}$, and then it experiences a rising trend afterward. The static refractive index along $x$ and $z$, respectively, confirms the metallic and semiconductor behaviors of the optical properties of the material although the refractive index value is less than 1 after the visible energy edge for the $x$ direction. Therefore, the superluminal phenomenon has occurred in all these areas (speed of electromagnetic waves is greater than the speed of light), and the very small amount of the refractive index (less than vacuum) is evidence of the transparency of this compound in the mentioned direction. However, a relatively large refractive index can be seen in the IR region and the energy range between 2.5 and 10.5 (of course in the range of semiconductor materials) and the superluminal phenomenon has also occurred after $10.5 \mathrm{eV}$.

Extinction coefficient of a material is a measure of the absorption of electromagnetic radiation by matter. In fact, if the material has a low extinction coefficient, an electromagnetic wave easily passes through. According to Fig. 7, the maximum peaks of the EllZ case at the energy of the $0.83 \mathrm{eV}$ the lowest transmission and maximum adsorptions occur.

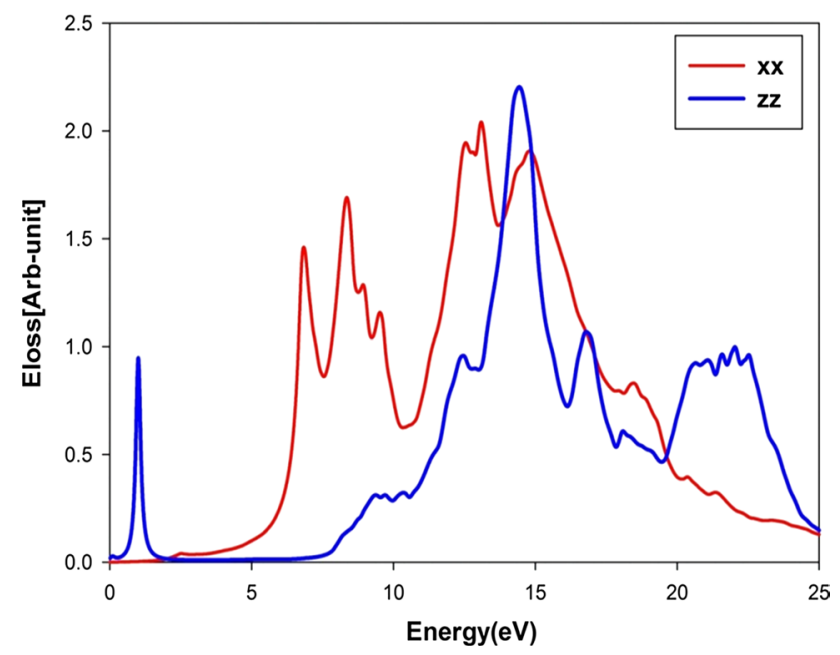

Fig. 6 E-loss function of pmmm boron along the $\mathrm{x}$ and $\mathrm{z}$ directions

Table 1 Maximum values of the imaginary part of the dielectric function and E-loss along the $\mathrm{x}$ and $\mathrm{z}$ directions

\begin{tabular}{llllllll}
\hline 18.14 & 16.82 & 14.43 & 12.43 & 10.31 & 9.35 & 1 & E loss z \\
18.14 & 16.5 & 12.95 & 10.57 & 10.04 & 8.56 & 0.78 & IM- EPSILON-Z \\
\hline 14.86 & 13.11 & 12.53 & 9.52 & 8.93 & 8.35 & 6.81 & E loss x \\
& 14.01 & 10.73 & 9.3 & 8.77 & 7.45 & 2.53 & IM- EPSILON-X \\
\hline
\end{tabular}



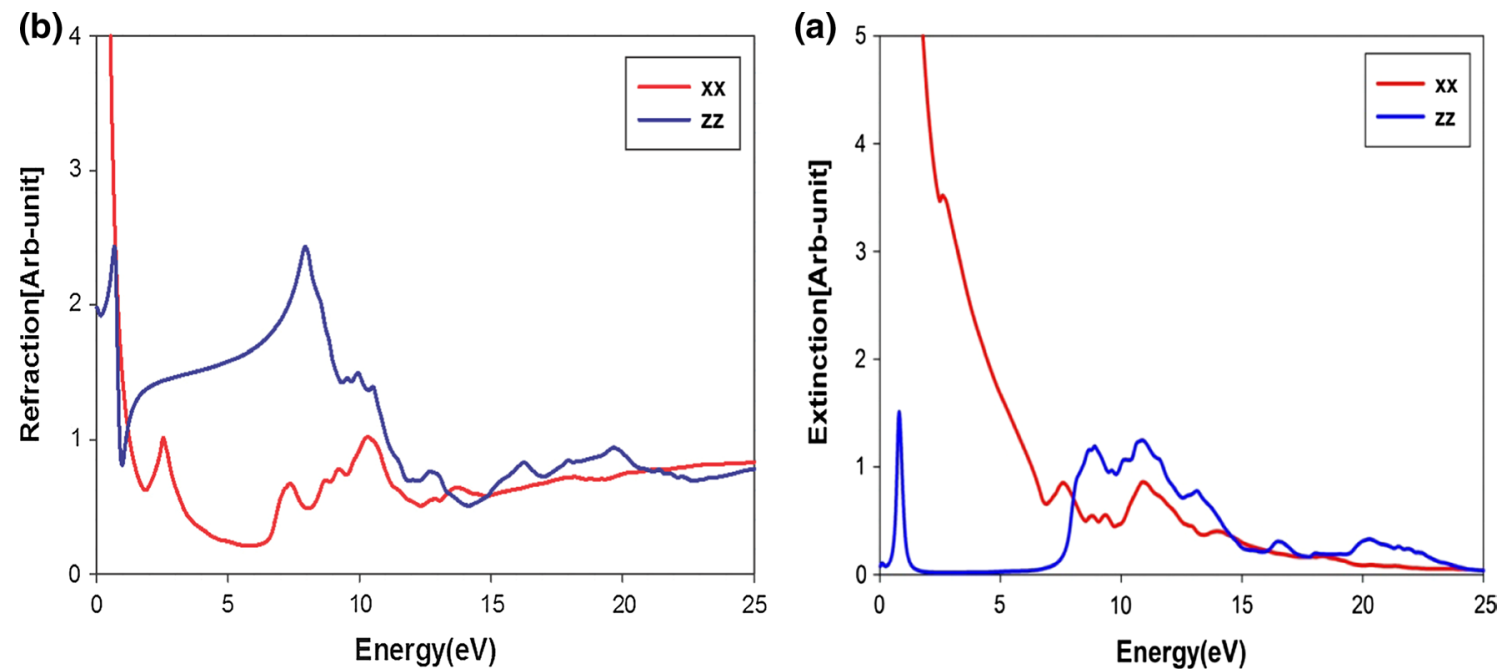

Fig. 7 The a real part and $\mathbf{b}$ imaginary part of the refractive index function for pmmm boron

The extinction coefficient is zero in the energy range from 1.5 to $6.5 \mathrm{eV}$ that can completely pass through. For the ElIX mode, the extinction coefficient decreases to reach its minimum value at $6.87 \mathrm{eV}$ which electromagnetic waves hardly pass through the material in the region before this energy. As shown in the diagram, there is anisotropy in the extinction coefficient at lower energies so that the decrease magnitude of the electromagnetic wave amplitude is high along $x$ and it is falling with a steep slope till $6.87 \mathrm{eV}$. On the contrary, the amplitude decrease and consequently absorption does not occur in the energy range from 0 to $7.5 \mathrm{eV}$, except for the edge of the visible region, which, of course, is confirmed by the absorption graph in Fig. 9.

According to Fig. 8, the maximum reflection for EllZ takes place at the energy of $0.76 \mathrm{eV}$, and it declines to reach zero at the energy of $1.15 \mathrm{eV}$. Then, after a rise in reflection, it tends to zero at the energies higher than $15 \mathrm{eV}$. In EllX mode, the reflection has a decreasing trend, reaching its minimum at $10 \mathrm{eV}$, and then it goes up a little while it tends toward zero after the energies above $15 \mathrm{eV}$ like the ElIZ one. It is observed that the optical properties vary at lower energies in different directions; for the EllX mode, the reflection is almost $100 \%$ at lower energies while for the EIIZ mode, the reflection is much less at the same energies and the electromagnetic waves pass through the material. This indicates directional-dependence of the optical properties of borophene whose reflections at lower energies for $x$ and $z$ axes are another testimony to the very strong metallic and semiconductor behaviors of this compound, respectively. Hence, on the one hand, the absorption and reflection are minimal in the $z$ direction, and on the other hand, the extinction coefficient is also low, so borophene is transparent. After $15 \mathrm{eV}$, the behavior is isotropic and the structure is transparent in both directions.

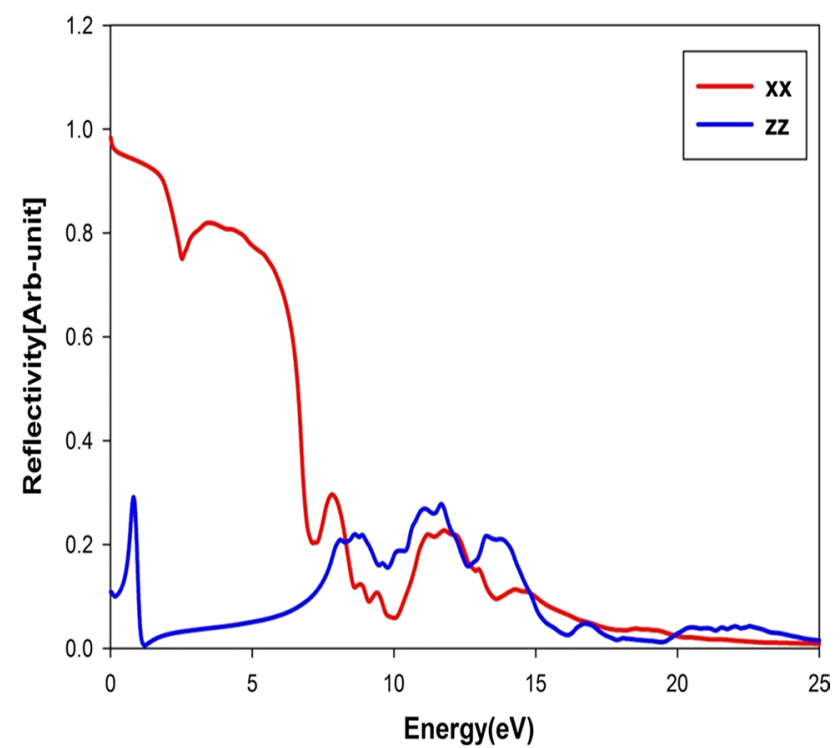

Fig. 8 The reflectivity for pmmm boron along the $x$ and $z$ directions

Optical absorption is the result of the interband and intraband transitions taking place from the occupied states to the unlocked ones. In the case of the absorption coefficient for the EIIZ state, according to Fig. 9, the absorption amount is very small at the energies less than $6.1 \mathrm{eV}$, except near $0.78 \mathrm{eV}$. Although the maximum absorption is at energies of $10.94 \mathrm{eV}$ and $8.98 \mathrm{eV}$, it decreases with fluctuations afterward. For ElIX, besides a peak at $10.89 \mathrm{eV}$, the maximum absorption occurs in visible region at the energies of $0.68 \mathrm{eV}$ and $2.79 \mathrm{eV}$, and the absorption decreases in this direction at higher energies. The anisotropy is clear owing to the difference in the absorption at lower energies, and it is the re-confirmation 


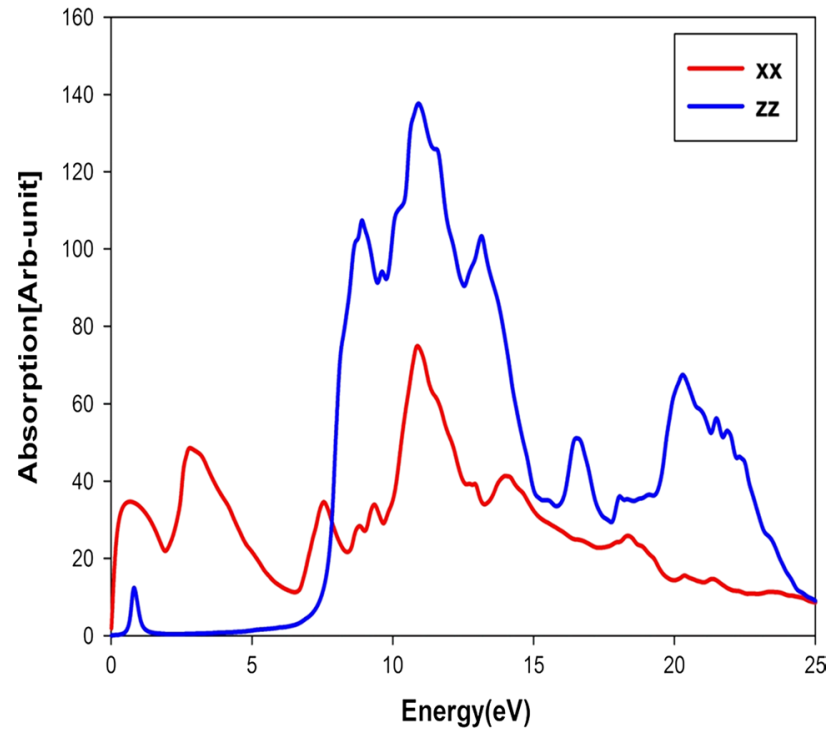

Fig. 9 The absorption coefficient for pmmm boron along the $x$ and $z$ directions

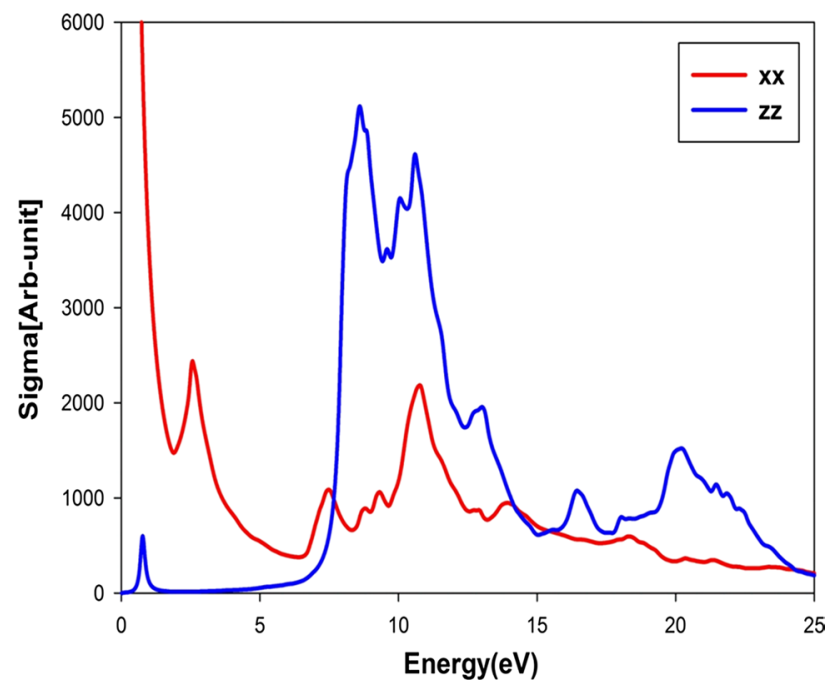

Fig. 10 Optical conductivity of pmmm boron along the $x$ and $z$ directions

of the metallic property and the insulating behavior along $x$ and $z$, respectively.

Optical conductivity is dependent on the imaginary part of the dielectric tensor where the electrons in occupied states are induced by the absorbing a photon into unoccupied states above Fermi level leading to the interband transition. In EIIZ mode (Fig. 10), it is observed that the optical conductivity begins after a gap at about $0.5 \mathrm{eV}$, and the amount of optical conductivity is also very small afterward in the visible area. Moreover, it reaches its maximum magnitude at energies of $8.62 \mathrm{eV}$ and $10.62 \mathrm{eV}$ supporting the insulating behavior in this direction discussed in the previous sections. Along the $\mathrm{x}$ axis, the conductivity decreases from its infinite amount which validates the metallic behavior in this direction, to reach its minimum value at the energy of $1.8 \mathrm{eV}$, and it experiences an elevation to reach the maximum amount at $2.58 \mathrm{eV}$. Then, it continues a downward trend after some fluctuations.

Due to the unique and interesting properties of twodimensional materials, these materials have been considered in recent years, and many researchers have studied their electronic and optical properties including graphene, germanene, silicene and stanene [34]. In this section, a comparison is made between the results of this work and others with the mentioned materials and borophene.

The real part of the dielectric function of borophene in the perpendicular direction to the sheet is greater than the values of this quantity in the other two-dimensional materials, and in the parallel axis, it is less than the reported values for other two-dimensional materials. The peak magnitudes of the energy loss function of the borophene in the vertical and parallel directions are greater than those in the germanene, silicene and stanene, and it is less than the graphene peak value. The borophene static refractive index in both directions is higher than those of the mentioned materials. In the case of borophene, like four other materials, the greatest reflection in the perpendicular direction occurs in the UV region while it is happening in IR and visible areas for the parallel direction. In the viewpoint of absorption, borophene is more similar to graphene.

Recently, with the recognition of the interesting and unique properties of two-dimensional materials, the application of these materials has been considered in the manufacture of electronic materials, including solar cells. For example, graphene, as the first known two-dimensional material, has a high potential for use in low-cost, flexible solar cells with high efficiency, which is due to the electron transport properties and the high mobility of charge carriers [35]. Phosphorene is also a known two-dimensional material and its application in solar cells has been studied by combining with other suitable materials as an electron donor or acceptor, as well as changing the properties to increase the efficiency of solar cells, such as impurities injections, order changes, number of phosphorene layers and applying the electric field [36-46]. With regard to the characteristics of borophene, including the amount of absorption, reflection and electrical conductivity, it appears that this material, such as other two-dimensional materials, can have an effective efficiency in increasing the solar cells' efficiencies.

\section{Conclusions}

In this paper, the optical and electronic properties of one type of borophene structures by pmmm space group is theoretically studied using the density functional theory with the 
Wien2k simulation package. Lattice parameters obtained are consistent with the reported values. According to the density of states and the band structure, it can be anticipated that this material has metallic properties while its bulk state shows semiconductor properties. The optical properties, including the real and imaginary parts of the dielectric function, the refractive index, the energy loss function, reflection, absorption and optical conductivity, are investigated and analyzed in two directions: perpendicular and parallel. Furthermore, the metallic and semiconductor properties have been seen in the parallel and perpendicular directions, respectively. While the optical properties are often different at lower energies for different directions, they are almost in line with each other at higher energies. With respect to the mentioned features of borophene, it appears to have a strong potential to escalate the efficiency of solar cells.

Open Access This article is distributed under the terms of the Creative Commons Attribution 4.0 International License (http://creativecommons.org/licenses/by/4.0/), which permits unrestricted use, distribution, and reproduction in any medium, provided you give appropriate credit to the original author(s) and the source, provide a link to the Creative Commons license, and indicate if changes were made.

\section{References}

1. Geim, A.K.: Graphene: status and prospects. Science 324(5934), 1530-1534 (2009)

2. Nair, R.R., et al.: Fine structure constant defines visual transparency of graphene. Science 320(5881), 1308 (2008)

3. Guzman-Verri, G.G., Voon, L.C.L.Y.: Electronic structure of silicon-based nanostructures. Phys Rev B 76(7), 075131 (2007)

4. Vogt, P., et al.: Silicene: compelling experimental evidence for graphene-like two-dimensional silicon. Phys Rev Lett 108(15), 155501 (2012)

5. Feng, B., et al.: Evidence of silicene in honeycomb structures of silicon on $\mathrm{Ag}(111)$. Nano Lett. 12(7), 3507 (2012)

6. Cahangirov, S., et al.: Two- and one-dimensional honeycomb structures of silicon and germanium. Phy s Rev Lett. 102(23), 236804 (2009)

7. Dávila, M.E., et al.: Germanene: a novel two-dimensional germanium allotrope akin to graphene and silicone. New J Phys 16(9), 095002 (2014)

8. Zhong, Q., et al.: Synthesis of borophene nanoribbons on $\mathrm{Ag}(110)$ surface. Phys Rev Mater 1, 021001 (2017)

9. Liu, C.-C., Jiang, H., Yao, Y.: Low-energy effective Hamiltonian involving spin-orbit coupling in silicene and two-dimensional germanium and tin. Phys Rev B 84, 19 (2011)

10. $\mathrm{Xu}, \mathrm{Y}$., et al.: Large-gap quantum spin hall insulators in tin films. Phys Rev Lett 111, 13 (2013)

11. Zhu, F., et al.: Epitaxial growth of two-dimensional stanene. Nat Mater. 14, 1020 (2015)

12. Albert, B., Hillebrecht, H.: Elementary challenge for experimenters and theoreticians. Angew Chem Int Ed. 48(46), 8640 (2009)

13. Sergeeva, A.P., et al.: Understanding boron through size-selected clusters: structure, chemical bonding, and fluxionality. Chem Res 47(4), 1349 (2014)

14. Oganov, A.R., et al.: Ionic high-pressure form of elemental boron. Nature 457(7231), 863 (2009)
15. Ogitsu, T., Schwegler, E., Galli, G.: $\beta$-Rhombohedral boron: at the crossroads of the chemistry of boron and the physics of frustration. Chem Rev 113(5), 3425 (2013)

16. Huang, W., et al.: A concentric planar doubly $\pi$-aromatic B19cluster. Nat Chem 2(3), 202 (2010)

17. Popov, I.A., et al.: A combined photoelectron spectroscopy and ab initio study of the quasi-planar B24-cluster. Chem Phys 139(14), 144307 (2013)

18. Piazza, Z.A., et al.: Planar hexagonal B36 as a potential basis for extended single atom layer boron sheets. Nat Commun. 5(1), 3113 (2013)

19. Penev, E.S., et al.: Polymorphism of two-dimensional boron. Nano Lett. 12(5), 2441 (2012)

20. $\mathrm{Wu}, \mathrm{X}$., et al.: Two-dimensional boron monolayer sheets. ACS Nano 6(8), 7443 (2012)

21. Liu, Y., Penev, E.S., Yakobson, B.I.: Probing the synthesis of twodimensional boron by first-principles computations. Angew Chem Int Ed. 52(11), 3156 (2013)

22. Mannix, A.J., et al.: Synthesis of borophenes: anisotropic, twodimensional boron polymorphs. Science 350(6267), 1513 (2015)

23. Feng, B., et al.: Synthesis of borophenes: anisotropic, two-dimensional boron sheets. Nat Chem 8(6), 563 (2016)

24. Ozdogan, C., et al.: The unusually stable B100 fullerene, structural transitions in boron nanostructures, and a comparative study of $\alpha$ - and $\gamma$-boron and sheets. Phys Chem C 114(10), 4362 (2010)

25. Wu, X., et al.: Two-dimensional boron monolayer sheets. ACS Nano 6(8), 7443 (2012)

26. Yu, X., et al.: Prediction of two-dimensional boron sheets by particle swarm optimization algorithm. Phys Chem C 116(37), 20075 (2012)

27. Ren, X., et al.: Random-phase approximation and its applications in computational chemistry and materials science. Mater Sci. 47(21), 7447 (2012)

28. Zhou, X., et al.: Semimetallic two-dimensional boron allotrope with massless dirac fermions pe with massless dirac fermions. Phys Rev Lett 112(8), 085502 (2014)

29. Peng, B., et al.: Stability and strength of atomically thin borophene from first principles calculations. Mater Res Lett 5(6), 399 (2017)

30. Penev, E.S., Kutana, A., Yakobson, B.I.: Can two-dimensional boron superconduct? Nano Lett. 16(14), 2522 (2016)

31. Feng, B., et al.: Dirac fermions in borophene. Phys Rev Lett. 118(9), 096401 (2017)

32. Wang, H., et al.: Strain effects on borophene: ideal strength, negative possion's ratio and phonon instability. New J Phys 18(7), 073016 (2016)

33. Liu, H., et al.: Phosphorene: an unexplored 2D semiconductor with a high hole mobility. ACS Nano 8(4), 4033 (2014)

34. John, R., Merlin, B.: Optical properties of graphene, silicene, germanene, and stanene from IR to far UV - a first principles study. Phys Chem Solids 110, 307 (2017)

35. Shamsudin, M.S., Sanip, S.M.: A review on the two-dimensional graphene exploited in organic solar cells applications. Adv Mater Res 1109, 514 (2015)

36. Zhang, C.H., Feng, Y.P., Shen, L., Phosphorene and transition metal dichalcogenide 2D heterojunctions: application in excitonic solar cells. arXiv: 1507.07343 (2015)

37. Dai, J., Zeng, X.C.: Bilayer phosphorene: effect of stacking order on bandgap and its potential applications in thin-film solar cells. Phys Chem Lett 5(7), 1289 (2014)

38. Zhou, L., et al.: Novel excitonic solar cells in phosphorene- $\mathrm{TiO}_{2}$ heterostructures with extraordinary charge separation efficiency. Phys Chem Lett 7(10), 1880 (2016)

39. Ţălu, Ştefan, et al.: Microstructure and tribological properties of FeNPs@a-C: H films by micromorphology analysis and fractal geometry. J Ind Eng Chem 43, 164-169 (2016) 
40. Ţălu, Ştefan, et al.: Microstructure and micromorphology of $\mathrm{Cu} /$ Co nanoparticles: surface texture analysis. Electron Mater Lett. 12(5), 580-588 (2016)

41. Boochani, Arash, et al.: Calculation of half-metal, debye and curie temperatures of $\mathrm{Co}_{2} \mathrm{VAl}$ compound: first principles study. Commun Theor Phys. 63(5), 641 (2015)

42. Majidi, Solaiman, et al.: Effect of point defects on the electronic density states of $\mathrm{SnC}$ nanosheets: first-principles calculations. Results in physics 7, 3209-3215 (2017)

43. Zare, Maryam, Solaymani, Shahram, Shafiekhani, Azizollah, Kulesza, Slawomir, Ţălu, Ştefan, Bramowicz, Miroslaw: Evolution of rough-surface geometry and crystalline structures of aligned $\mathrm{TiO}_{2}$ nanotubes for photoelectrochemical water splitting. Sci Rep 8(1), 10870 (2018)

44. Ţălu, Ştefan, Bramowicz, Miroslaw, Kulesza, Slawomir, Dalouji, Vali, Solaymani, Shahram, Valedbagi, Shahoo: Fractal features of carbon-nickel composite thin films. Microsc Res Tech 79(12), 1208-1213 (2016)

45. Ţălu, Ştefan, Bramowicz, Miroslaw, Kulesza, Slawomir, Ghaderi, Atefeh, Dalouji, Vali, Solaymani, Shahram, Khalaj, Zahra: Microstructure and micromorphology of $\mathrm{Cu} / \mathrm{Co}$ nanoparticles: surface texture analysis. Electron Mater Lett. 12(5), 580-588 (2016)

46. Ţălu, Ştefan, et al.: Microstructure and tribological properties of FeNPs@a-C: H films by micromorphology analysis and fractal geometry. J Ind Eng Chem. 43, 164-169 (2016)

Publisher's Note Springer Nature remains neutral with regard to jurisdictional claims in published maps and institutional affiliations. 